\title{
MRM; making DAM and CRM stick together: The ten functional areas of marketing
}

\section{Romek Jansen}

worked as a marketing manager for an international publisher and a business intelligence software company, after a career in sports marketing. Nowadays, he works as a business consultant, helping companies to maximize their operational marketing efficiency. Romek worked on the design and implementation of an ordering/procurement/distribution solution for Hewlett Packard's "Operation One Voice" during its merger with Compaq.

\section{Frans Riemersma}

worked as a consultant and successfully delivered MRM projects for HP EMEA, Unilever and Adidas global, after a career in internet and mobile marketing. As a consultant, Frans helps marketing services departments of large companies, supporting them from organizational change to implementation of web applications, intranets, extranets, websites, mobile marketing services, interactive television and IVR solutions.

Keywords: digital asset management, marketing resource management, marketing efficiency, marketing complexity, marketing operations, 10 functional areas of marketing

Abstract The marketing function is becoming more complex. Marketers are trying to launch more campaigns in shorter timeframes addressing more target audiences. In the process of bringing your marketing message to the consumer, you need many different processes, skill sets supported by a variety of software, ranging from DAM to $\mathrm{CRM}$, including also ERP and BI. With these facts in mind, we discuss a model called the " 10 functional areas of marketing." This model helps marketers to create an integrated marketing environment that performs better, faster and cheaper. Vice versa, the model helps software vendors to create their own roadmap for new functionality.

Journal of Digital Asset Management (2007) 3, 65-70. doi:10.1057/palgrave.dam.3650066

Romek Jansen MRMlogiQ,

1e Oosterparkstraat 219 Amsterdam $1091 \mathrm{HA}$

The Netherlands

Tel: $+31(0) 643047311$ E-mails: info@mrmlogiq. com, www.mrmlogiq.com, RomekJansen@mrmlogiq com

\section{“HOW DOES DAM RELATE TO MRM?"}

As marketing efficiency consultants and implementers of Marketing Resource

Management (MRM) software solutions, we have increasingly received questions about how DAM relates to MRM. Where DAM is focussed on controlling everything around your content elements, where CRM tells you where it is most likely to find a customer, MRM links the two together and helps you to create, produce and distribute marketing material within time and within budget.

In the process of bringing your marketing message to the consumer, you need to create copy and images, you need to publish these digital assets in a certain format, you need to procure print or media space and you need to distribute the message. This involves many different processes, skill sets supported by a variety of software, ranging from DAM to CRM, including also ERP and BI.

The challenge marketing managers face today is to integrate all these different areas within the marketing department. For companies this results into a quicker time to market of campaigns, decreased costs for print orders, increased consistency of marketing material, etc.

We have created a model that helps marketers increasing marketing efficiency by designing a roadmap for integrated marketing. We identified and described the ten functional areas of marketing. As an additional result, software vendors explained us that the same model helped them to create their own roadmap for adding new functionality to their software. 
In this paper, we highlight DAM within the MRM space and show how you can create an overview covering all those different areas in marketing.

We will describe and explain the model of "ten functional areas" in this paper and show how CRM and DAM will eventually meet. We will discuss some high-level trends that we have seen over the last decades that make marketing environments increasingly complex. Seemingly small changes can have a major impact on the manageability of marketing. You will see complexity is "exploding" at this moment. Using commonly accepted communication models as a starting point, we provide a framework for our MRM model of ten functional areas.

\section{THE ORIGIN OF MARKETING COMPLEXITY}

Every day managers go to work, trying to change the future of their organizations. Most are doing absolutely the best they can under given circumstances. They are trying to hang in there, firefighting, lobbying, wrestling through that pile of e-mails while attempting in the meantime to make their planning for the coming months. If you look at it marketers are launching more campaigns in shorter timeframes addressing more target audiences. Will they be able to keep up or do we need a drastic and different approach? The answer is in the legend of the Persian King.

"Hundreds and hundreds of years ago there was a King in Persia who loved to play games. But he had gotten bored of the games that were present at the time and wanted a new game that was much more challenging. He commissioned a poor mathematician who lived in his kingdom to come up with a new game."

"After months of struggling with all kinds of ideas, the mathematician came up with the game of Chaturanga. The game had two armies, each led by a King who commanded his army to defeat the other by capturing the enemy King. It was played on a simple $8 \times 8$ square board."

"The King loved this game so much that he offered to give the poor mathematician anything he wished for. 'I would like one grain of rice for the first square of the board, two grains for the second, four grains for the third and so on, doubled for each of the 64 squares of the game board' said the mathematician. 'Is that all?' asked the King, 'Why don't you ask for gold or silver coins instead of rice grains'. 'The rice should be sufficient for me', replied the mathematician."

"The King ordered his staff to lay down the grains of rice. Unfortunately for him, he had committed to deliver $\left(2^{64}-1\right)$ grains of rice $18,446,744,073,709,551,615$ to be exact - a quantity somewhat larger than the volume of the planet Earth. In fact the whole kingdoms supply of rice was exhausted before the 40th square was reached."

"The Persian King was beaten without seeing it coming. Shah-mat, chess mate, game over!"

If you think of it, marketers resemble the Persian King. Do marketers gamble a hand full of rice or their whole kingdom? Complexity in marketing communications has always been hard to capture. Like some primitive cultures that had only five numbers, counting 1,2,3,4 followed by "many," marketing situations are just "complex." No numbers, no reasons, having no clue about operational consequences of seemingly small decisions in the company. After 4 it seems like we stop counting.

\section{BACK TO BASICS}

Do you still remember your early days in marketing communications? 10 to 1 your first class or course started out with the famous model by Shannon \& Weaver, Maletzke or the one from Lasswell. The latter described the very basics of communication in 1948 as: "Who? Says what? In what channel? To whom? To what effect?" Or to put it in differently: SenderMessage-Channel-Receiver-Effect. This model was originally designed to explain the information flow with regards to public speaking. A sender creates a message, which is transported through a medium, which intentionally will be perceived by the receiver, to finally result in the awaited effect. This shows the most important components on how information exchange becomes effective communication. 
Numerous communication scientists have created their own version or variation on this principle, all with their specific twists and focuses, but they all consist of the following elements; Source-Message-Channel-Receiver, often abbreviated as the SMCR Model.

- Source: the entity wishing to present a particular view of event or object

- Message: signal or combination of signals that form a body of information

- Channel: vehicle, medium or carrier through which signals are sent

- Receiver: the target, person or thing, that takes in messages

Although these early communication models definitely had their fair share of critiques, mainly focused on their simplicity not entirely reflecting reality, we would like to use this simple SMCR model to "eliminate the unnecessary so that the necessary may speak."

The SMCR model was originally designed as a so-called "transmission model" that describes single information transactions. It is, however, also very helpful when describing the functional areas that exist in the so-called "end-to-end process" of bringing a message to the market.

- The Source: the brand owner

- The Message: the digital assets

- The Channel: the media (both ATL and BTL)

- The Receiver: the consumer

As a brand owner you need to create digital assets, you need to produce material that is suitable for the media in your strategy and you need to distribute these marketing materials to your potential customers.

With market segmentation creating complexity on the receiver side and product diversification creating complexity on the source side, the amount of communication transactions grows rapidly. There are more audiences to talk to and more things to talk about. Now, in theory, marketers can utilize cross-media and multi-channel communications to offer "tailor made solutions" to individuals. Although new technologies can be used to automate some manual activities, these new media are often based on different technology platforms, communicating different messages using different formats, putting even more pressure on the marketing department.

\section{THE EVOLUTION OF MARKETING}

The incredible speed with which the current marketing environment changes, definitely leads to certain problems:

- Migration from a mass market to a highly segmented market, potentially 1-to-1

- Existence of more products in more variation with shorter product life cycles, potentially heading into the direction of "tailor made solutions"

- Communicating different kinds of information

- Communicating via an increasing amount of interactive media

All of this means, the amount of information transactions not just increase, but "explode." If you are a brand owner, bringing ten new products to the market every six months, using ten different media (eg packaging, product manual, sales guide, website, in store display, etc) to communicate to four different segments of the market, in ten languages, you are talking about $20 \times 10 \times 4 \times 10=8,000$ different publications a year!

As in every evolutionary process (eg biological, technological), growth in information transactions occurs exponentially. Every year the amount of data stored doubles. Even if innovation in technologies can keep up with the speed, people need to revise their skills in order to adapt to the changing circumstances and processes need to be adjusted to changing volumes. Marketers definitely experience the fall-back of this growth. It is impossible for individuals to keep up with this information expansion. Managers have the feeling that they have less control over what "goes out," they suffer more stress and are heading for "Information Fatigue Syndrome," the 21st century burn out.

The sheer magnitude of this information growth and the requirements on how to accommodate these volumes is highly underestimated by many people. This psychological phenomenon originates from the fact that people experience change, but it is perceived as a linear growth. Even an exponential curve approximates a straight line when viewed 
for a brief duration. Our intuition tells us that situations will change with the current pace.

Technology can be a great help, but will not offer the overall solution. Implementing a content management system, for instance, does not mean that you have content. The obvious conclusion is that we need to develop an environment that can adapt to rapid change and creates overall visibility and control. MRM offers a helping hand.

\section{THE FIVE MARKETING RESOURCES}

The challenge we face today is how to reengineer processes and create a marketing environment that can deal with contemporary and emerging market requirements.

MRM is all about the implementation of an efficient marketing environment. Successes are not only born out of brilliant ideas. Just as important is a brilliant execution of those ideas. MRM is an integrated methodology that orchestrates the most optimal use of your marketing resources, in order to achieve an effective implementation of your strategy. Ask yourself the question

"what needs to be created, by whom, using which technology, for which budget, in which timeframe?"

In our projects, we look at the five marketing resources that can be extracted from this question.

Materials: the output of the marketing process

- ads

- brochures

- DM campaigns

- website, etc.

Men/Women: all human resource-related assets

- knowledge

- FTE's

- roles \& responsibilities, etc.

Machines: technological infrastructure

- content management systems

- automated publishing module

- campaign management tool

- CRM, etc.
Money: Budgets and financial infrastructure

- cost centres

- procurement

- VAT

- cross billing, etc.

Minutes: Turn around times

- time to market

- deadlines, etc.

On a high level, Money and Minutes are the areas where you want to be more efficient, marketing strategies cause Materials to increase and Men and Machines is the battleground for middle management ... and your MRM strategy is going to be the glue.

\section{THE TEN FUNCTIONAL AREAS OF MRM}

MRM deals with the organization and orchestration of these five marketing resources, both externally as internally. Externally, it deals with the performance of suppliers. Internally, it obviously deals with the performance of the marketing department, but also with neighboring disciplines such as procurement, finance and ICT.

From initial concept through to contact with a customer or channel partner across the end-to-end process - several operational activities take place. These activities can be clustered in functional areas, subsets that require specific skill sets, knowledge and technological solutions. At a high level there are three broad categories based on the need to create, produce and distribute marketing materials. Drilling down, these can be broken out into ten different areas. Each single area can be optimized in itself, but "the win is in the integration." For each market, each corporate strategy and marketing strategy, you will find that a different set of functional areas are important.

Again, we took the SMCR model as a starting point. Logically, each of the processes of daily marketing activities supports information transactions from one of the four elements to another element in the SMCR model.

Therefore, on a high level we look at three 


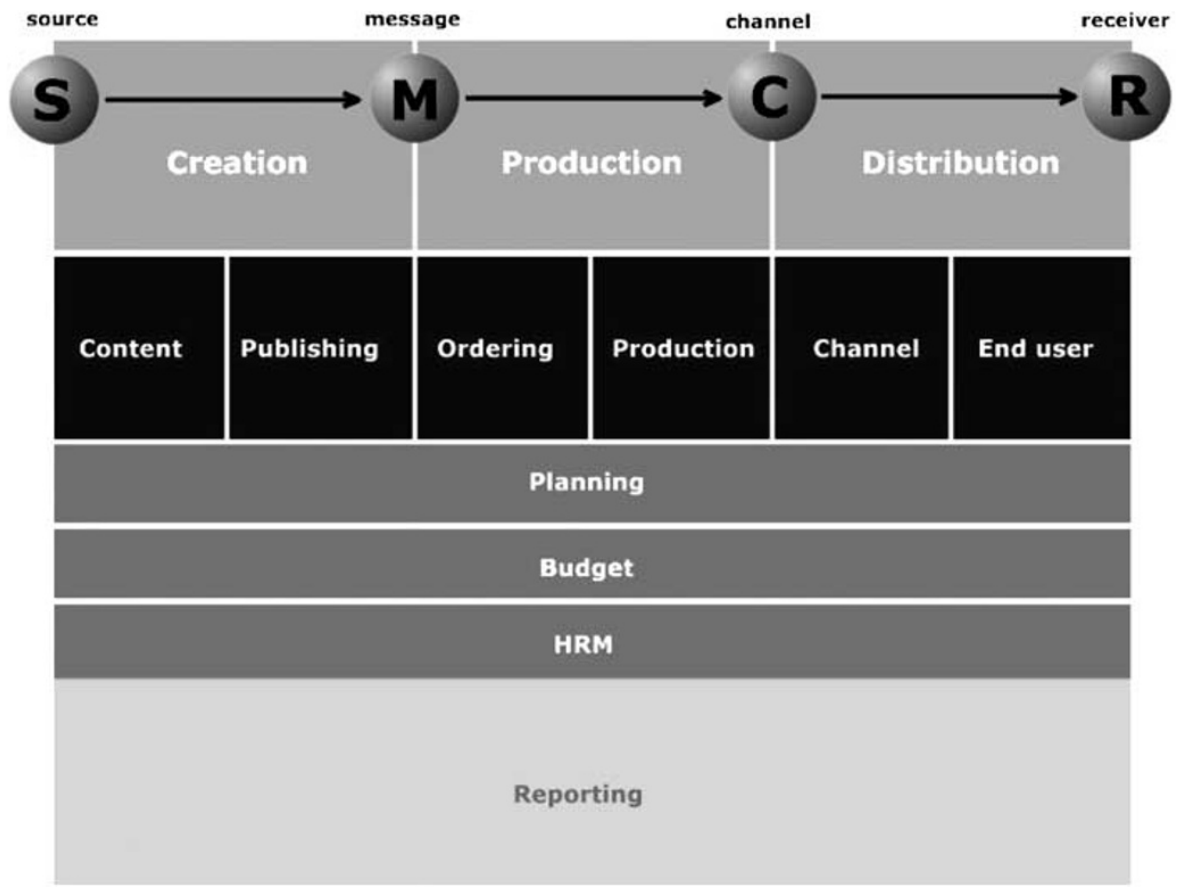

Figure 1: Ten Functional areas framework. (C MRMlogiQ 2004-2007

main processes: Creation, Production and Distribution of marketing material (Figure 1). You can see that DAM is covering mainly the Creative process (the two areas of content and publishing). Each of these main processes can be split up into two functional areas. The main processes together form what we call the endto-end process of marketing, or the primary process of marketing.

The secondary process supports the end-toend process. This process keeps track of time, budget and human resources. In order to see what the trends are in your marketing environment a third layer, the reporting layer, should keep track of data regarding the other nine functional areas, like for example time spent, content created, items produced, storage costs, etc.

1. Content: Creating, managing and storing images, texts, logos, audio- \& video files

2. Publishing: Templates, placeholders, brand guides used to combine content elements and prepare it for publication/ production

3. Ordering: Capturing "demand" for the production of marketing material
4. Production: Procurement and production of the actual material

5. Channel: Distributing the (trade)marketing material via a channel partner

6. End user: Distributing the (consumer) marketing material directly to a potential customer

7. Planning: Keeping track of deliverables and deadlines from $\mathrm{A}$ to $\mathrm{Z}$ on a program or project level like a campaign

8. Budget: Keeping track of spending from A to $\mathrm{Z}$ on a program or project level

9. HRM: Keeping track of knowledge, documentation, roles and responsibilities in processes and user management in software

10. Reporting: Keeping track of marketing operations (all nine other areas) using reports, KPI's and dashboards.

\section{FUTURE OF DAM}

If you are a DAM software vendor, you will have experienced increasing competition from vendors who traditionally used to operate outside the DAM arena. ERP, CRM and even BI software vendors have started integrating DAM functionalities to complete their marketing 


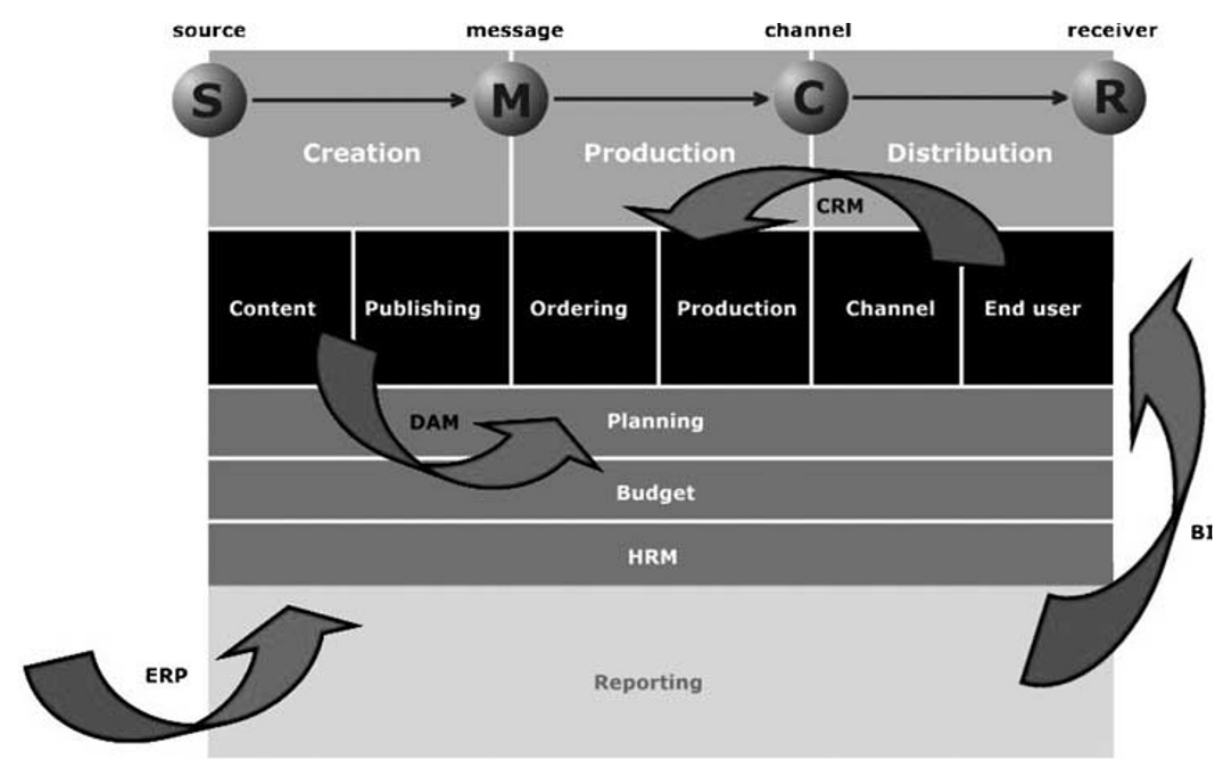

Figure 2: ERP, CRM and BI players moving in ( $2004-2007$ MRMlogiO

software tool. By looking at the arrows drawn in the ten functional areas framework (Figure 2), you will see which players are moving into which area.

If you have a CRM solution in place, you like to obtain the most out of your market segmentation by only communicating relevant information, potentially 1 -to- 1 to your prospects or clients. Because of the huge volumes involved, an automated publishing module that generates emails, brochures or proposals is a logical next step. Your CRM partner will be happy to look into this business opportunity.

ERP system providers are contented to extent functionality with some marketing-specific requirements. BI vendors come up with standardized marketing intelligence modules that offer basic CRM functionalities. MRM software vendors who already have some kind of DAM tool functionality, are expanding and professionalizing the depth of the content repositories.

The same trend can, however, be seen from a DAM perspective. Some DAM suppliers, for example, have started to add automated publishing modules, collaboration tools or even project planning tools to their DAM solution. They have started to add more functional areas to their software. Any time soon they will meet, for example, ERP and CRM software vendors.

Whether the big will eat the small, the fast will eat the slow, the cheap will eat the expensive or the smart will eat the stupid, is hard to say. Scalability, flexibility and usability of the systems will definitely play a major role. Whatever happens, whoever wins, at the end a smiling marketing manager will be there to handover the flowers. 